DOI: http://doi.org/10.21857/yvjrdcnqpy

\title{
SOME EXTENSIONS OF THE NOTION OF LOOP GRASSMANNIANS
}

\author{
IVAN MiRKOVIĆ
}

To Sibe Mardešić

\begin{abstract}
We report an ongoing attempt to establish in algebraic geometry certain analogues of topological ideas, The main goal is to associate to a scheme $X$ over a commutative ring $\mathbb{k}$ its "relative motivic homology" which is again an algebro geometric object over the base $\mathbb{k}$. This is motivated by Number Theory, so the Poincare duality for this relative motivic homology should be an algebro geometric incarnation of Class Field Theory.
\end{abstract}

\section{INTRODUCTION}

1.1. A geometric view on Number Theory. We propose an algebro geometric framework for the basic organizational principles in Number Theory framework, the Class Field Theory and its nonabelian version, the Langlands conjectures. The setting for this will be Geometric Representation Theory, the strategy of encoding algebraic problems into algebro geometric objects.

This paper is actually twice removed from Number Theory itself. First, I restrict to the baby case of Number Theory where the field $\mathbb{Q}$ of rational numbers is replaced by its geometric analogue, the field of rational functions on a curve defined over a finite field. At present geometric method works the best in this case. Second, the number theoretic interest is primarily in the "topological" aspect of schemes, i.e., one works in étale topology (or Dmodules). However, we will use Zariski topology and strive towards a "proper" Zariski grounding for the "topological" questions.

After these two drastic simplifications something is still left as one can try to extend the experience of number theory to general algebro geometric objects (curves can be replaced by algebraic varieties and finite fields with

2010 Mathematics Subject Classification. 11S37, 14F42.

Key words and phrases. Loop Grassmannian, class field theory, motivic homology. 
general commutative rings). The classical results of this type are the AbelJacobi construction of Jacobians, the canonical self duality of Jacobians and the Contou-Carrère symbol.

In this setting Class Field Theory appears to be concerned with the Poincare duality for a certain "relative motivic homology" in algebraic geometry (section 2). In the abelian case my optimistic attitude is that the shape of this program is "reasonably" clear. ${ }^{(1)}$ Now, the content of Langlands conjectures should be a nonabelian version of Poincare duality. However, in this nonabelian case such formulation is not clear in dimension one and even the nature of the basic objects is presently not known beyond dimension one.

1.2. Loop Grassmannians. From the present geometric point of view a loop Grassmannian $\mathcal{G}(G)$ of a group $G$ is the homology of a formal one dimensional disc with coefficients in $G$. The exposition here will be concerned with the modifications of the notion of loop Grassmannians. From the Number Theory point of view a loop Grassmannian is a geometrization of Hecke operators and for physicists this is a realization of 't Hooft operators in QFT.

Loop Grassmannians $\mathcal{G}(G)$ of algebraic groups $G$ are recalled in section 3 , together with a generalization, the loop Grassmannians $\mathcal{G}(G, Y)$ with a "condition $Y$ " where $Y$ is a $G$ space with a chosen point $y$. Here $\mathcal{G}(G, Y)$ consists of elements of $\mathcal{G}(G)$ whose "singularity gets resolved in $Y$ ". Here $\mathcal{G}(G, Y)$ and $\mathcal{G}(G)$ are general cohomological constructions and $\mathcal{G}(G, Y)$ is used in order to systematically deal with the geometry of interesting subspaces of $\mathcal{G}(G)$.

Section 4 presents another generalization of loop Grassmannians. From the data of a set $I$, a quadratic form $Q$ on $\mathbb{Z}[I]$ and a poset $P$, one produces "loop Grassmannian" $\mathcal{G}^{P}(I, Q)$. This in particular provides a "semi-infinite" reconstruction of ordinary loop Grassmannians based on finite subschemes of a curve.

Section 5 is a homage to the Kamnitzer-Knutson conjecture on a relation of loop Grassmannians and quivers. This still open conjecture was an inspiration for the work in section 4 .

Finally, section 6 lists the attempts towards constructing loop Grassmannians in arbitrary dimension.

1.2.1. Some roots of this project. My background is in Geometric Representation Theory. At its height this field bridges the chasm between the Langlands program in Number Theory and the Quantum Field Theory in physics. At an infinity of the present project lies its goal, a transparent algebro-geometric foundation for these developments, uniform across various cases and dimensions.

\footnotetext{
${ }^{1}$ For the 1-dimensional Number Theory the étale Class Field Theory has been given a Poincare duality interpretation by Mazur. I do not yet understand the relation with the present geometric Class Field Theory.
} 
One "new" ingredient here is the notion of local spaces which is a version of the Beilinson-Drinfeld notion of factorization spaces. I have formulated it at the time of the paper [11] as a formalization of the Finkelberg-Schechtman work [4] on quantum groups and its version [11] on semi-infinite loop Grassmannians. However, this notion first became useful when I tried to understand the Kamnitzer-Knutson conjecture on the relation of quivers and loop Grassmannians [12].

1.2.2. Acknowledgments. For me Sibe Mardešić is a friendly and beautiful presence in my life, an enthusiast for mathematics and for life. His deep impact on me came from my impression that Sibe was able to make the right decisions in all aspects of his life with others.

Some parts of this project are with Yaping Yang and Gufang Zhao as indicated below. I have received much help from many people. In particular some key ideas here were only made possible by extensive discussions with Sam Raskin and Nick Rozenblyum. Zhijie Dong has found numerous errors and several counter-examples for my conjectures. This text also uses the notes of Raeez Lorgat on my lectures at the Schrödinger Institute in Wien (January 2017).

This work has been partially supported by NSF grants, a sabbatical semester from U. of Massachusetts at Amherst and the Simons Fellowship that extended the sabbatical semester to a full year. Some key breakthroughs occurred during visits to two mathematical institutions. In Fall semester of 2014 I was at the Mathematical Sciences Research Institute in Berkeley during their Geometric Representation Theory program (funded by MSRI as a Simons Research Professor and as an Eisenbud Research Professor). I spent February of 2015 at University of Chicago (funded by their Mathematics Department).

\section{Geometric Class Field Theory}

The Geometric Class Field Theory presently exists only in dimension one. Here we find a way to restate the known results in a way which is uniform in local and global situations and suggests a generalization to arbitrary dimension.

\subsection{Homology as free abelian group.}

2.1.1. Free abelian group $A_{X}$ generated by $X$. In a given setting $\mathcal{S}$ for a space $X \in \mathcal{S}$ we denote by $S_{X}$ the free commutative monoid ("semigroup") object generated by $X$ in $\mathcal{S}$ (or in a given larger class $\widetilde{\mathcal{S}}$ ). Also, $A_{X}$ denotes the free abelian group object generated by $X$.

In sets: $S_{X}=\mathbb{N}[X]$ and $A_{X}=\mathbb{Z}[X]$. In categories $S_{\emptyset}=A_{\emptyset}$ is a point while $S_{\mathrm{pt}}=(\mathcal{F} \mathcal{S}, \sqcup)$ is the tensor category of finite sets with disjoint union. This is related to exponentiation since for a groupoid category $\mathcal{C}$ the size gets exponentiated: $\left|A_{\mathcal{C}}\right|=e^{|\mathcal{C}|}$. In the setting of categories $A_{\mathrm{pt}}$ is in some sense the sphere spectrum. 
In the homotopy category of pointed topological spaces, $S_{X}$ is the infinite symmetric power of $X$ while $A_{X}$ is its group completion. By the Dold-Thom theorem this $A_{X}$ is essentially the total homology $H_{*}(X, \mathbb{Z})$. More precisely $H_{i}(X, \mathbb{Z})=\pi_{i}\left(A_{X}\right)$ is a truncation of the group object $A_{X}$.

2.1.2. Free abelian group $A_{X}$ as homology of $X$. The above Dold-Thom theorem says that in the homotopy category of pointed topological spaces the free abelian group $A_{X}$ is a "derived" version of homology. This is intuitively clear from the defining property $\operatorname{Hom}\left(A_{X}, \mathcal{A}\right)=\operatorname{Map}(X, \mathcal{A})$ of $A_{X}$. In a nonderived setting the right hand side is $H^{0}(X, \mathcal{A})$ but when we work in a derived setting it becomes the (derived version of) the cohomology $H^{*}(X, \mathcal{A})$. So, the derived $A_{X}$ satisfies the property of homology that the $\mathcal{A}$-dual of homology is the cohomology with coefficients in $\mathcal{A}$.

Suslin has imitated the Dold-Thom mechanism in algebraic geometry, his incarnation of homology is the sheaf with transfers $\mathbb{Z}_{X}^{t r}$. This was extended by Voevodsky to a construction of motivic (co)homology. We will follow the same idea but we will take a slightly different route towards constructing a "relative motivic homology" which is expected to be a simplification of motivic homology useful for Geometric Class Field Theory.

2.2. The local geometric Class Field Theory of Contou-Carrère.

2.2.1. $A_{X}$ for ind-finite indschemes. Over a given commutative ring $\mathbb{k}$ we consider the setting $S c h_{\mathbb{k}}^{f}$ of finite $\mathbb{k}$-schemes and the class $\mathrm{C}$ of affine commutative group indschemes. For a finite scheme $F$ over $\mathbb{k}$ we are interested in $A_{F} \in \mathrm{C}$, the object of $\mathrm{C}$ freely generated by $F$.

Lemma. $A_{F}$ exists and it is given by the "double dual"

$$
\operatorname{Hom}_{\mathrm{C}}\left[\operatorname{Map}_{S c h_{\mathrm{k}}}\left(F, G_{m}\right), G_{m}\right] \text {. }
$$

Proof. The key is that the Cartier duality operation $\mathbb{D}=\operatorname{Hom}_{\mathrm{C}}\left(-, G_{m}\right)$ on $\mathrm{C}$ is involutive. The double dual construction satisfies the defining property of $A_{F}$ since for any $\mathcal{A} \in \mathrm{C}$

$$
\begin{gathered}
\operatorname{Hom}_{\mathrm{C}}\left[\mathbb{D}\left(\operatorname{Map}_{S c h_{\mathrm{k}}}\left(F, G_{m}\right)\right), \mathcal{A}\right]=\operatorname{Hom}_{\mathrm{C}}\left[\mathbb{D} \mathcal{A}, \operatorname{Map}_{S c h_{\mathrm{k}}}\left(F, G_{m}\right)\right] \\
=\operatorname{Map}_{S c h_{\mathrm{k}}}\left(F, \operatorname{Hom}_{C}\left[\mathbb{D} \mathcal{A}, G_{m}\right]\right)=\operatorname{Map}_{S c h_{\mathrm{k}}}(F, \mathcal{A}) .
\end{gathered}
$$

REmarks. (0) The lemma automatically extends to ind-finite indschemes $F$.

(1) Group $A_{F}$ can be called multiplicative distributions on $F$. If we replace $G_{m}$ by $G_{a}$ we would find that the ordinary distributions on $F$ form the vector space generated by $F$. 
2.2.2. $\underline{\mathcal{G}}\left(G_{m}\right)$ is the group indscheme $A_{d}$ generated by a disc $d$. For a smooth curve $C$ Hilbert schemes of points $\mathcal{H}_{C}^{n}$ are the same as symmetric powers $S^{n} C$. This is a monoid for the operation of addition of divisors. In terms of the ideal $\mathcal{I}_{D}=\mathcal{O}_{C}(-D)$ of a subscheme $D \subseteq C$ this is the tensoring of line bundles: $\mathcal{I}_{D^{\prime}+D^{\prime \prime}}=\mathcal{I}_{D^{\prime}} \otimes_{\mathcal{O}_{C}} \mathcal{I}_{D^{\prime \prime}}$.

Consider the formal disk $d=\widehat{c}$ at a point $c \in C$. This is an ind-finite indscheme.

Corollary. The canonical map $S_{d} \rightarrow A_{d}$ is the same as the Abel-Jacobi map

$$
S^{\bullet} d=\mathcal{H}^{\bullet} d \stackrel{\iota}{\hookrightarrow} \mathcal{G}\left(G_{m}\right) \text { for } \quad \iota(D) \stackrel{\text { def }}{=} \mathcal{O}_{d}(-D) .
$$

Proof. Any map $f: d \rightarrow \mathcal{A}$ with $\mathcal{A}$ a commutative monoid in indschemes extends canonically to a homomorphism of monoids $F: \mathcal{H}_{d} \rightarrow \mathcal{A}$, the value at a finite subscheme $D$ is the integral of $f$ over $D$. Therefore $\mathcal{H}_{d}=S_{d}$.

Since the reduced part $d_{\text {red }}$ of $d$ is a single point $c$, the reduced parts of $S_{d} \subseteq A_{d}$ are $\mathbb{N} c \subseteq \mathbb{Z} c$. The points of $\mathcal{H}^{\bullet} d$ over a $\mathbb{k}$-ring $\mathbb{k}^{\prime}$ are the monic polynomials $z^{n}+a_{1} z^{n-1}+\cdots+a_{0}$ with all $a_{i} \in \mathbb{k}^{\prime}$ nilpotent.

Group $\left(G_{m}\right)_{\mathcal{K}}$ is the product of 4 subgroups $G_{m}$ (the constant loops in $\left.G_{m}\right), \mathbb{Z}$ (loops that are powers of $z$ ) and of the positive and negative congruence subgroups $\left(K_{+}\right)\left(\mathbb{k}^{\prime}\right)=1+z \mathbb{k}^{\prime}[[z]]$ and $\left(K_{-}\right)\left(\mathbb{k}^{\prime}\right)=1+z^{-1} \mathcal{N}_{\mathbb{k}^{\prime}}\left[z^{-1}\right]$ where $\mathcal{N}_{\mathbb{k}^{\prime}} \subseteq \mathbb{k}^{\prime}$ is the ideal of nilpotent elements [6]. Now, $\underline{\mathcal{G}}\left(G_{m}\right)=\left(G_{m}\right)_{\mathcal{K}} /\left(G_{m}\right)_{\mathcal{O}}=$ $z^{\mathbb{Z}} \times K_{-}$. This is the same as what one obtains from $\left(\mathcal{H}_{d},+\right)$ by inverting a single element, the center $c$ of the disc (when one views $\mathcal{H}^{\bullet} d$ in terms of monic polynomials this means inverting the equation $z$ of $c$ ).

Corollary. The identification $A_{d} \cong \mathcal{G}\left(G_{m}\right)$ is a Poincare duality in algebraic geometry.

"Proof". Poincare Duality is the identification of homology and the compactly supported cohomology. We have argued above that $A_{d}$ is the value of a certain homology on $d$. We have identified $A_{d}$ with $\underline{\mathcal{G}}\left(G_{m}\right)$ which is by definition the local cohomology of the disc at the point $\bar{c} \in d$ of $G_{m}[1]$. However, due to the nature of $d$ (when one removes $c$ one removes all compact subschemes of $d$ ), this is also the compactly supported cohomology of $d$.

Finally, we saw that the isomorphism $A_{d} \stackrel{\cong}{\longrightarrow}\left(G_{m}\right)$ is generated by the Abel-Jacobi map $d \rightarrow \mathcal{G}\left(G_{m}\right), x \mapsto \mathcal{O}_{d}(-x)$. This can be interpreted as the use of the diagonal $\Delta_{d}$ in $d \times d_{c}$ where $d_{c}$ is obtained by embedding $d$ into a smooth compact curve and then collapsing the complement of the point $c$. This indeed fits into the Poincare Duality pattern.

2.3. An axiomatic setting for relative motivic cohomology. Let $S c h_{\mathbb{k}}$ denote the $\mathbb{k}$-schemes of finite type. The meaning of the abelian group generated by a $\mathbb{k}$-scheme $X \in S c h_{\mathbb{k}}$ depends on the class of groups ("the setting") that one considers. Here we axiomatize the necessary properties for such settings. 
2.3.1. A setting C for constructing $A_{X}^{\mathrm{C}}$. Here, C should be an infinity category of commutative group objects in higher algebraic $\mathbb{k}$-stacks, with the following structures

(1) compatible inner Hom functors

$$
S c h_{\mathbb{k}}^{o} \times \mathrm{C} \stackrel{\text { Map }}{\longrightarrow} \mathrm{C} \text { and } \mathrm{C}^{o} \times \mathrm{C} \stackrel{\mathcal{H o m}}{\longrightarrow} \mathrm{C}
$$

(2) a dualizing object $\mathcal{D} \in \mathrm{C}$, i.e., we ask that the functor $\mathbb{D} \stackrel{\text { def }}{=} \mathcal{H o m}(-, \mathcal{D}): \mathrm{C} \rightarrow \mathrm{C}$ is an involution on $\mathrm{C}$.

Once we have such setting one can form the corresponding free abelian groups just as in the case of finite schemes:

"LEMMA". For $X \in S c h_{\mathbb{k}}$ there exists an object $A_{X}^{\mathrm{C}}$ of $\mathrm{C}$ freely generated by $X$. This is

$$
\mathcal{H o m}[\operatorname{Map}(X, \mathcal{D}), \mathcal{D}]=\mathbb{D}[\mathcal{M a p}(X, \mathcal{D})] \text {. }
$$

REMARKs. (1) Having a dualizing object $\mathcal{D}$ guarantees existence of $A_{X}^{\mathrm{C}}$ which is the C-version of homology.

It also gives a reasonable cohomology theory $H^{*}(-, \mathcal{D})$. The Poincare Duality for this cohomology theory is then a natural isomorphism of $A_{X}^{\mathrm{C}}$ with $H_{c}^{*}(X, \mathcal{D})$.

(2) In reality we have an increasing sequence of categories $C_{n}$ (for us these will be the groups in $\leq n$-stacks over $\mathbb{k}$ ), with the corresponding duality objects (we will use $G_{m}[n]$ ). Then all these $A_{X}^{n}=A_{X}^{\mathrm{C}_{n}}$ form a system $A_{X}$ which is the homology that we are interested in. (An example of truncations of this type, i.e., as $A_{X}^{n}$ truncates $A_{X}$, is the Deligne cohomology.)

ExAmPLE. Let $C$ be a complete smooth connected curve with the Picard scheme $\operatorname{Pic}(C)$. Then the system $A_{C}^{n}$ stabilizes at $n=1$ and the only nontrivial map is the truncation to the connected components

$$
A_{X}^{1}=\operatorname{Bun}_{G_{m}}(C)=\operatorname{Pic}(C) / G_{m} \rightarrow A_{X}^{0}=\mathbb{Z} .
$$

2.4. Relative motivic cohomology conjecture.

2.4.1. Conjecture. In the above setting there exists a cohomology theory for $\mathbb{k}$-schemes with values in commutative group objects in higher algebraic $\mathbb{k}$-stacks, such that

1. Its homology $\mathbb{H}_{*}(X, \mathbb{Z})$ is $A_{X}^{\bullet}$.

2. It has the four functors functoriality: $f_{*}, f_{!}$and $f^{*}, f^{!}$.

3. For smooth schemes it satisfies Poincare duality.

ThEOREM. True in dimension $\leq 1$.

There are more expectations: 
(a) This should be the relative version of the (diagonal part of) motivic cohomology in the sense that the value on a $\mathbb{k}$-scheme should be the $\mathbb{k}$-representable part of the diagonal motivic cohomology. (The usual motivic cohomology is a group in sets and when one can make it into a functor in $\mathbb{k}$-algebras this functor need not be representable in algebraic geometry over $\mathbb{k}$.)

This version should be easier than the original. In the original construction the geometric cycles are irreducible varieties which are not allowed to degenerate to schemes. The conjectural construction axiomatized above would be more direct with fewer restrictions, for instance it should have a local theory and a Cartier duality.

One should also be able to reconstruct $A_{X}^{\bullet}$ as in Voevodsky theory, using the " $k$-relative" analogue of the category of finite correspondence (based on the construction $\mathbb{Z}_{X}^{\text {loc }}$ in 4.1.2).

(b) Its Poincare duality should be the geometric CFT (at present only known for curves). This may opens a door to higher geometric Langlands.

(c) The "theory of higher adeles" deals with a certain formally open cover of a scheme, a scheme has Grothendieck's "universal" stratification by generic points of irreducible subschemes and it generates a formally open cover by formal neighborhoods of such generic points The relative motivic homology $A_{X}^{\bullet}$ should be computable in terms of this cover.

2.4.2. Example: Relative Milnor K-theory of higher local fields. Motivic cohomology is understood in weight one (essentially dimension one) and for fields. For a field $\mathfrak{K}$ one can describe the diagonal part of motivic cohomology algebraically as its Milnor K-theory

$$
K_{*}^{\mathcal{M}}(\mathfrak{K}) \stackrel{\text { def }}{=} T_{\mathbb{Z}}^{\bullet}\left(\mathfrak{K}^{*}\right) /\langle\text { Steinberg relation }\rangle .
$$

If $\mathbb{k}$ is a field then the $n$-dimensional local field $\mathcal{K}_{n}=\mathbb{k}\left(\left(x_{1}\right)\right) \cdots\left(\left(x_{n}\right)\right)$ has itself a structure of an indscheme over $\mathbb{k}$. Now we define its relative (to $\mathbb{k}$ ) Milnor K-theory $K_{i}^{\mathcal{M}}\left(\mathcal{K}_{n} / \mathbb{k}\right)$ by the same formula but now calculated in the tensor category $\left(C, \otimes_{C}\right)$ of commutative affine group indschemes over $\mathbb{k}$. We define this tensor structure as the Cartier dual of bilinear maps into $G_{m}$ :

$$
A \otimes_{\mathrm{C}} B \stackrel{\text { def }}{=} \mathbb{D}\left[\mathcal{B} i l_{A, B}\left(G_{m}\right)\right] .
$$

Now the diagonal part of the Poincare duality for $\operatorname{Spec}\left(\mathcal{K}_{n}\right)$ in relative motivic cohomology should be the following.

Conjecture (with Nick Rozenblyum). The corresponding 2 dimensional Contou-Carrère (see [14]), gives Cartier duality of $K_{i}^{\mathcal{M}}\left(\mathcal{K}_{n} / \mathbb{k}\right)$ and $K_{j}^{\mathcal{M}}\left(\mathcal{K}_{n} / \mathbb{k}\right)$ for $i+j=n+1$. 
Remarks. (1) For $n=1$ this is the local geometric Class Field Theory of Contou-Carrère. In general. $A_{\operatorname{Spec}\left(\mathcal{K}_{n}\right) / \mathbb{k}}$ should be $K_{n}^{\mathcal{M}}\left(\mathcal{K}_{n} / \mathbb{k}\right)$.

(2) Here, it is essential that our tensor product satisfies an unusual property that $G_{m} \otimes_{c} G_{m}=0$. The duality is also not true for the original Milnor K-theory of $\mathcal{K}_{n}$ which is "too large" ([14]).

\section{Loop Grassmannians $\mathcal{G}(G)$ in Drinfeld's Language of Finitely SUPPORTED MAPS.}

Here we recall the loop Grassmannians $\mathcal{G}(G)$ of algebraic groups $G$. We consider a generalization, the loop Grassmannians $\mathcal{G}(G, Y)$ with a "condition $Y$ " where $Y$ is a $G$ space with a chosen point $y$. This is a sub-indscheme of $\mathcal{G}(G)$ consisting of objects whose "singularity gets resolved in $Y$ ".

\subsection{Loop Grassmannians as partial flag varieties.}

3.1.1. The standard loop Grassmannians $\mathcal{G}(G)$. Let $\mathbb{k}$ be a commutative ring and let $\mathcal{O}=\mathbb{k}[[z]] \subseteq \mathcal{K}=\mathbb{k}((z))$ be the Taylor and Laurent series over $\mathbb{k}$. For an algebraic group scheme $G$ denote by $G_{\mathcal{O}} \subseteq G_{\mathcal{K}}$ its disc group scheme and loop group indscheme over $\mathbb{k}$ whose points over a $\mathbb{k}$-algebra $\mathbb{k}^{\prime}$ are $G_{\mathcal{O}}\left(\mathbb{k}^{\prime}\right)=G\left(\mathbb{k}^{\prime}[[z]]\right)$ and $G_{\mathcal{K}}\left(\mathbb{k}^{\prime}\right)=G\left(\mathbb{k}^{\prime}((z))\right)$. The standard loop Grassmannian is the ind-scheme given by the quotient in the fpqc topology

$$
\underline{\mathcal{G}}(G)=G_{\mathcal{K}} / G_{\mathcal{O}} .
$$

When $G$ is reductive then $\underline{\mathcal{G}}(G)$ is a partial flag variety of the corresponding Kac-Moody group $\widehat{G}$.

ExAmple. The loop Grassmannian of $G=G L(V)$ is the space of lattices in $V_{\mathcal{K}}$, i.e., the union of all

$$
\mathcal{L}_{p, q} \stackrel{\text { def }}{=}\left\{z^{p} V_{\mathcal{O}} \subseteq L \subseteq z^{-q} V_{\mathcal{O}} ; L \text { is an } \mathcal{O} \text {-submodule }\right\}, \quad p, q \in \mathbb{N} .
$$

Taking the quotient by the lower bound this is seen as the $z$-fixed part $G r\left(z^{-q} V_{\mathcal{O}} / z^{p} V_{\mathcal{O}}\right)^{z}$ of a finite dimensional Grassmannian. We see for $G=$ $G L(V)$ the general fact that the loop Grassmannian $\mathcal{G}(G)$ is an ind-system of finite dimensional schemes (and these schemes are proper for reductive groups $G)$.

Subexample. In this realization one can compute. Say, $\mathcal{L}_{-1,0}$ is $G r(V) \stackrel{\text { def }}{=} \sqcup_{p} G r_{p}(V)$. For $\operatorname{dim}(V)=2$ this decomposition of $\mathcal{L}_{-2,0}$ has terms for $0 \leq p \leq 4$ and the reduced part of these are

$$
\left[\mathcal{L}_{-2,0}\right]_{\text {red }} \cong \mathrm{pt} \sqcup \mathbb{P}^{1} \sqcup \overline{T \mathbb{P}^{1}} \sqcup \mathbb{P}^{1} \sqcup \mathrm{pt} .
$$

Here, the middle term is the one point compactification of the tangent bundle. 
3.1.2. Parameterization of orbits in $\mathcal{G}(G)$. Let $G$ be reductive with a Borel subgroup then let $B=N \ltimes T$ and let $W$ be the corresponding Weyl group, the quotient $N_{G}(T) / T$ of the normalizer of the Cartan $T$.

Notice that the coweights, i.e., the cocharacters $X_{*}(T)$ of the Cartan subgroup embed into $\mathcal{G}(T)$ by restricting a cocharacter $\lambda: G_{m} \rightarrow T$ to the punctured formal neighborhood of 0 in $\mathbb{A}^{1}$. For $\lambda \in X_{*}(T)$ we will also denote by $\lambda$ the corresponding point in $\mathcal{G}(T) \subseteq \mathcal{G}(G)$.

The partial flag variety $\mathcal{G}(G)$ has several kinds of Schubert cell decompositions given by the orbits of the Iwahori subgroup $I \subseteq G_{\mathcal{O}}$, the negative Iwahori subgroup $I^{-} \subseteq G_{\mathbb{k}\left[z^{-1}\right]}$ and the loop group $N_{\mathcal{K}}$ (and by certain mixtures of these). All these classes of orbits are parameterized by $X_{*}(T) \subseteq \mathcal{G}(G)$.

3.1.3. Powers of a space. One way to develop a homology theory is to start with a given notion of powers of a space $M$. Besides the Cartesian powers $M^{n}$ we also have symmetric versions and comparison maps

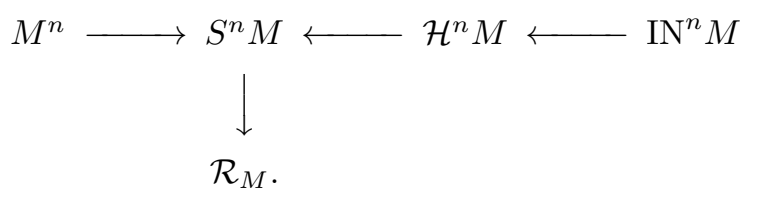

(1) A symmetric power is a categorical quotient $S^{n}=M^{(n)} \stackrel{\text { def }}{=} M^{n} / / S_{n}$.

(2) A Hilbert power $\mathcal{H}_{M}^{n}=M^{[n]}$ is the principal irreducible component of the moduli $\mathrm{Hilb}^{n} M$ of length $n$ subschemes of $M$ (the closure of the submoduli of discrete subschemes).

(3) An Ito-Nakamura power $\operatorname{IN}^{n} M$ is the principal irreducible component of the moduli of $S_{n}$-clusters in $M$, i.e., $S_{n}$-invariant subschemes $\mathcal{D}$ of $M$ such that $\mathcal{O}(D)$ is a regular representation of $S_{n}$.

(4) The Ran space $\mathcal{R}_{M}$ which is the moduli of all finite subsets of $M$. This is a certain ind-system of powers $M^{n}$ where the maps in the systems kill the ordering of points and their multiplicities. It is a notion of an infinite power of a space.

Remark. Any map of $M$ into a group extends to $M^{n}$. If the group is commutative this factors through $S^{n} M$.

3.1.4. Cohomology: The Beilinson-Drinfeld loop Grassmannian. For a finite subset $E$ of a smooth curve $C$, the first $G$-cohomology of $C$ with the support at $E$ is the moduli $H_{E}^{1}(C, G)$ of pairs $(\mathcal{T}, \tau)$ of a $G$-torsor $\mathcal{T}$ over $C$ and its section (i.e., trivialization) $\tau$ over $C-E$. Notice that this is also the moduli of maps of pairs $\operatorname{Map}[(C, C-E),(\mathbb{B}(G), \mathrm{pt})]$ (a map from $C$ to the classifying space $\mathbb{B}(G)$ is a $G$-torsor $\mathcal{T}$ over $C$ while the compatibility of the map $C \stackrel{\mathcal{T}}{\longrightarrow} \mathbb{B}(G)$ with the canonical map $C-E \rightarrow$ pt is a trivialization $\tau$ of $\mathcal{T}$ over $C-E$.) 
As $E$ varies in the Ran space $\mathcal{R}_{C}$ one assembles the spaces

$$
\mathcal{G}(G)_{E} \stackrel{\text { def }}{=} H_{E}^{1}(C, G)=\operatorname{Map}[(C, C-E),(\mathbb{B}(G), \mathrm{pt})]
$$

into an ind-scheme $\mathcal{G}(G)=\mathcal{G}_{C}(G)$ over $\mathcal{R}_{C}$ called the Beilinson-Drinfeld loop Grassmannian.

LEMMA. A choice of a local coordinate $z$ on the formal neighborhood $\widehat{c}$ of a point $c \in C$ gives an isomorphism of the simplest fiber with the standard loop Grassmannian

$$
\underline{\mathcal{G}}(G) \stackrel{\cong}{\longrightarrow} \mathcal{G}(G)_{c} \text {. }
$$

Proof. The map $G_{\mathcal{K}} \rightarrow H_{c}^{1}(\widehat{c}, G)$ by $g \mapsto(G \times \widehat{c}, g)$ is surjective since any $G$-torsor on $\widehat{c}$ is trivial, It factors to an isomorphism $\underline{\mathcal{G}}(G) \stackrel{\cong}{\longrightarrow} H_{c}^{1}(\widehat{c}, G)$ because the group $G_{\mathcal{K}}$ is the moduli of triples $\left(\mathcal{T}, \tau, \tau_{\text {in }}\right)$ of a $G$-torsor $\mathcal{T}$ on $C$ and sections $\tau, \tau_{\text {in }}$ of $\mathcal{T}$ over $\widehat{c}-c$ and $\widehat{c}$.

Finally, the restriction from $C$ to $\widehat{c}$ gives $H_{c}^{1}(C, G) \stackrel{\cong}{\longrightarrow} H_{c}^{1}(\widehat{c}, G)$.

3.2. Loop Grassmannians $\mathcal{G}(G, Y)$ with a condition $Y$. Drinfeld noticed that a number of important constructions are moduli of maps of pairs. This is used here to define a kind of cohomology with an "extra condition". This is just a formalization of Drinfeld's description of zastava spaces.

For any $G$-space $Y$ with a point $y$, the "loop Grassmannian with the condition $(Y, y)$ " is the space $\mathcal{G}(G, Y)$ above $\mathcal{R}_{C}$ with the fiber at $E \in \mathcal{R}_{C}$ given by

$$
\mathcal{G}(G, Y)_{E} \stackrel{\text { def }}{=} \operatorname{Map}[(C, C-E),(Y / G, \mathrm{pt})] .
$$

LEMMA. $\mathcal{Y}=\mathcal{G}(G, Y)$ is a factorization space, i.e., there is a canonical and consistent system of identifications of fibers at disjoint $E^{\prime}, E^{\prime \prime} \in \mathcal{R}_{C}$,

$$
\mathcal{Y}_{E^{\prime}} \times \mathcal{Y}_{E^{\prime}} \stackrel{\cong}{\longrightarrow} \mathcal{Y}_{E^{\prime} \sqcup E^{\prime \prime}}
$$

ExAmple. [The Beilinson-Drinfeld fusion] As different points $a, b \in C$ approach a single point $c$ the pair $\{a, b\}$ approaches in $\mathcal{R}_{C}$ the singleton $\{c\}$. Therefore, $\mathcal{G}(G, Y)_{a} \times \mathcal{G}(G, Y)_{b}=\mathcal{G}(G, Y)_{\{a, b\}}$ approaches $\mathcal{G}(G, Y)_{c}$ in the total space of $\mathcal{G}(G)$. This is actually an ind-flat degeneration of $\mathcal{G}(G)^{2}$ to $\mathcal{G}(G)$, i.e., it is flat on finite dimensional pieces.

SubeXAmple. For $G=G L_{2}$, the product $\mathbb{P}^{1} \times \mathbb{P}^{1} \subseteq \mathcal{G}(G)_{a, b}$ converges to $\overline{T \mathbb{P}^{1}} \subseteq \mathcal{G}(G)_{c}$. (This happens inside a $\mathbb{P}^{3}$-bundle - a smooth quadric $x y=u v$ degenerates to a singular quadric $x y=u^{2}$.)

3.2.1. Usefulness of $\mathcal{G}(G, Y)$. Each $\mathcal{G}(G, Y)$ is a subfunctor of $\mathcal{G}(G, \mathrm{pt})=$ $\mathcal{G}(G)$ and a pair $(\mathcal{T}, \tau)$ in $\mathcal{G}(G)_{E}$ lies in $\mathcal{G}(G, Y)_{E}$ iff the singularities of the section $\tau$ at $E$ is "no worse then what $Y$ allows".

Moreover, if $Y$ is a separated scheme then $\mathcal{G}(G, Y)=\mathcal{G}(G, \overline{G \cdot y})$, so the only relevant case is when the orbit $G \cdot y$ is dense in $Y$. 
The interesting subschemes of $\mathcal{G}(G)$ such as orbits, closures of orbits and intersections of such are of the form $\mathcal{G}(G, Y)$. This allows performing traditional calculations on $\mathcal{G}(G)$ in terms of finite dimensional objects $Y$. Here are some elementary properties.

$G_{i}$ then

LEMma. (a) [Fibered products] If $G$ is a quotient ${ }^{(2)}$ of one of the groups

$$
\mathcal{G}\left(G_{1}, Y_{1}\right) \times_{\mathcal{G}(G, Y)} \mathcal{G}\left(G_{2}, Y_{2}\right) \cong \mathcal{G}\left(G_{1} \times{ }_{G} G_{2}, Y_{1} \times{ }_{Y} \times Y_{2}\right) .
$$

For example the conjunction of conditions in $\mathcal{G}(G)$ is given by the product of condition spaces: $\cap \mathcal{G}\left(G, Y_{i}\right)=\mathcal{G}\left(G, \prod Y_{i}\right)$.

(b) [Subgroups] For $K \subseteq G$ we have an inclusion of functors $\mathcal{G}(K) \subseteq \mathcal{G}(G)$. There is a restriction statement

$$
\mathcal{G}(G, Y) \cap \mathcal{G}(K)=\mathcal{G}(K, Y)=\mathcal{G}(K, \overline{K \cdot y})
$$

and an induction statement

$$
\mathcal{G}\left(K, Y^{\prime}\right)=\mathcal{G}\left(G, G \times{ }_{K} Y^{\prime}\right) \subseteq \mathcal{G}\left[G,\left(G \times{ }_{K} Y^{\prime}\right)^{a f f}\right] \subseteq \mathcal{G}(G) .
$$

For instance, for a Cartan $T$ in a reductive $G$ the $T$-fixed points are $\mathcal{G}(G)^{T}=$ $\mathcal{G}(T)$. Therefore $\mathcal{G}(G, Y)^{T}=\mathcal{G}(T, \overline{T \cdot y})$.

(c) [Symmetries] Let $A \subseteq G$ be the stabilizer of $y \in Y$ and let $\dot{A}$ be any subgroup of the normalizer $N_{G}(A)$ whose action on $G / A$ extends to $Y$. Then the group $\ddot{A} \stackrel{\text { def }}{=} A_{\mathcal{K}} \dot{A}_{\mathcal{O}} \subseteq G_{\mathcal{K}}$ preserves $\mathcal{G}(G, Y) \subseteq \mathcal{G}(G)$.

3.2.2. Example: Vinberg semigroups. The usual Vinberg semigroup is defined for semisimple groups with $\pi_{1} G=0$. For any homogeneous space $G / A$ which is quasi-affine (open in an affine variety) we define its Vinberg semigroup as the double centralizer

$$
\overline{\mathcal{V}}_{A}=\operatorname{End}_{\mathcal{Z}_{A}}\left[(G / A)^{\text {aff }}\right] \text { for } \mathcal{Z}_{A} \stackrel{\text { def }}{=} \operatorname{Aut}_{G \times N_{G}(A)}(G / A) .
$$

Let $\mathcal{V}_{A}$ be its group part. We will only consider the case when $G$ is reductive, then let $B=N \ltimes T$ be a Borel subgroup and let $\check{B}=\check{T} \check{N}$ be the corresponding data for the Langlands dual group $\check{G}$.

Theorem. (a) $G / A$ is quasi-affine whenever $A$ is a normal subgroup of a parabolic $P$, and lies between the unipotent radical $U$ of $P$ and the derived subgroup $P^{\prime}$ of $P$.

(b) When $\pi_{1} G=0$ the usual Vinberg semigroup of $G$ equals $\overline{\mathcal{V}}_{N}$.

(c) The intersection cohomology of $\mathcal{G}\left(\mathcal{V}_{N}, \overline{\mathcal{V}}_{N}\right]$ is the ring of functions on the "base affine space" of the Langlands dual group:

$$
I C\left[\mathcal{G}\left(\mathcal{V}_{N}, \overline{\mathcal{V}}_{N}\right]=\mathcal{O}(\check{G} / \check{N}) .\right.
$$

The irreducible components of $\mathcal{G}(N, G / B)$ form a natural basis of the enveloping algebra $U\left(\check{\mathfrak{n}}_{\mathbb{Z}}\right)$ for the Lie algebra $\check{\mathfrak{n}}$ of $\check{N}$.

\footnotetext{
${ }^{2}$ In the general case of maps $G_{i} \rightarrow G$ the formula is just a bit more complicated.
} 
(d) If $G$ is adjoint so that it has the wonderful compactification $\bar{G}$ then

$$
\underline{\mathcal{G}}(G, \bar{G})_{\text {red }}=\sqcup_{\lambda \in X_{*}(T) / W} G_{\mathcal{O}} \cdot \lambda .
$$

REMARK. For any semigroup closure $\bar{G}$ of $G$ the geometric Satake formalism with $\mathcal{G}(G, \bar{G})$ instead of $\mathcal{G}(G)$, produces a semigroup $\bar{G}^{\vee}$. For example, $\left(\overline{\mathcal{V}}_{G, N}\right)^{\vee}=\overline{\mathcal{V}}_{\breve{G}, \check{N}}$. Under certain nondegeneracy conditions this is a bijection of semigroup closures of $G$ and $\check{G}$.

\section{ReAssembling $\mathcal{G}(G)$ FROM INFINItesimals AND GENERALizATIONS$$
\mathcal{G}^{P}(I, Q)
$$

This is an extension of the construction from [12]. For a set $I$, a quadratic form $Q$ on $\mathbb{Z}[I]$ and a poset $P$ it produces spaces $Z^{P}(I, Q)$ ("zastava space"), $S^{P}(I, Q)$ ("semi-infinite space"), $\mathcal{G}^{P}(I, Q)$ ("loop Grassmannian"). When the data correspond to a semisimple adjoint algebraic group $G$ the last object is the usual loop Grassmannian $\mathcal{G}(G)$ reconstructed from the point of view of its finite dimensional pieces. (These "pieces" are intersections of closures of orbits of loop groups $N_{\mathcal{K}}^{ \pm}$in $\mathcal{G}(G)$ for unipotent radicals $N^{ \pm}$of two opposite Borel subgroups of $G$. These orbits are two opposite semi-infinite stratifications from 3.1.2.)

4.0.1. Data. From the point of view of a semisimple adjoint algebraic group $G$ the set $I$ is the set of simple coroots. It encodes the Cartan $T$ of $G$ as $\left(G_{m}\right)^{I}$ and it provides an auxiliary polarization - a direction in which the loop Grassmannian is grown from finite dimensional pieces related to finite schemes. The quadratic form $Q$ is in this case the basic level of $G$ (up to a diagonal shift).

For the reconstruction of $\mathcal{G}(G)$ the poset $P$ is a point. However, when $P$ is $[m]=\{1<\cdots<m\}$ we get the version $\mathcal{G}^{[m]}(G)$ of $\mathcal{G}(G)$ which is the natural space for realizing the level $m$ representations of the affine Lie algebra, this reproduces for positive level $m$ the analogues of the "magical" properties of level one basic representations. The motivation for introducing $[m]$ in the present setting is an outgrowth of the Kamnitzer-Knutson work on quiver Grassmannians.

4.0.2. "Remember infinitesimals". The geometry underlying a loop Grassmannians $\underline{\mathcal{G}}(G)$ is that of a formal disc $d$ which is itself an ind-system of finite subschemes. In this section we describe $\underline{\mathcal{G}}(G)$ in terms of the Hilbert scheme $\mathcal{H}_{d}=\mathcal{H}^{\bullet} d$, the moduli of these finite subschemes.

In the commutative case, the relation is transparent as $\mathcal{G}\left(G_{m}\right)$ is the group generated by the semigroup $\mathcal{H}_{d}$. For this reason the presentation of the loop Grassmannian $\mathcal{G}(G)$ of a reductive group in terms of infinitesimals will take the form of a close relation of $\mathcal{G}(G)$ to $\mathcal{G}(T)$ for the Cartan $T$ of $G$. Poetically, we recover $\mathcal{G}(G)$ by following the accumulation of "dust" (the infinitesimals) 
that produces $\mathcal{G}(T)$ and we give it a desired shape of $\mathcal{G}(G)$ by adding a little "water" (a quadratic form) to glue the dust.

REMARK. This idea of remembering with a space $M$ its relation to finite schemes ("infinitesimals") appears as "rigid" geometry in p-adic theory and as "semi-infinite" constructions for loop groups. This is also implicitly present in Hodge theory. In characteristic zero the $n^{\text {th }}$ piece of the Hodge filtration $F_{n} \mathcal{D}_{X} \subseteq \mathcal{D}_{X}$ on the sheaf of differential operators comes from the $n^{\text {th }}$ infinitesimal neighborhood of the diagonal $\Delta_{X} \subseteq X^{2}$. For a scheme $X$ over a finite field $\mathbb{F}$ the mixed sheaves on $X$ reflect the system of approximations of $X$ by finite sets of points over finite extensions of $\mathbb{F}$.

\subsection{Local spaces.}

4.1.1. Colored local spaces over a scheme. For a set $I$ we will call $\mathcal{H}_{X \times I} \cong$ $\left(\mathcal{H}_{X}\right)^{I}$ the $I$-colored Hilbert scheme of points for a scheme $X$. It is a disjoint union of moduli $\mathcal{H}_{X \times I}^{\alpha} \cong \prod_{i \in I} \mathcal{H}^{\alpha_{i}} X$ of subschemes of degree $\alpha \in \mathbb{N}[I]$. For a space $Z$ over $\mathcal{H}_{X \times I}$ we denote the fiber at $D \in \mathcal{H}_{X \times I}^{\alpha}$ by $Z_{D}$ and $Z^{\alpha}$ is the restriction to $\mathcal{H}_{X \times I}^{\alpha}$.

An $I$-colored local space $Z$ over a scheme $X$ is a space $Z \rightarrow \mathcal{H}_{X \times I}$ over the $I$-colored Hilbert scheme of points of $X$, together with a system of consistent isomorphisms for disjoint $D^{\prime}, D^{\prime \prime} \in \mathcal{H}_{X \times I}$

$$
\iota_{D^{\prime}, D^{\prime \prime}}: Z_{D^{\prime}} \times Z_{D^{\prime \prime}} \stackrel{\cong}{\longrightarrow} Z_{D^{\prime} \sqcup D^{\prime \prime}} .
$$

ExAmples. (0) For $a \in X$ and $i \in I$ we will call the fiber $Z_{a i}$ at $a i \in X \times I$ the $i$-particle at $a$. The regular part $\mathcal{H}_{X \times I}^{r e g}$ of $\mathcal{H}_{X \times I}$ consists of all $D=\left(D_{i}\right)_{i \in I}$ such that subschemes $D_{i} \subseteq X$ are discrete and disjoint. The locality reduces the regular fibers to products of fibers of particles: $Z_{D} \cong \prod_{a i \in D} Z_{a i}$. The remaining fibers are then viewed as collisions of particles. So, when $Z \rightarrow \mathcal{H}_{C}$ is flat all fibers are degenerations of products of particles.

(1) A weakly local space is when the structure maps $\iota$ are only embeddings. Any weakly local space $Z$ has its local part $Z^{\text {loc } \subseteq} Z$ which is the least local subspace of $Z$ that contains all particles. So, at $D \in \mathcal{H}_{X \times I}^{r e g}$ the fiber is $Z_{D}^{\text {loc }}=\prod_{a i \in D} Z_{a i}$ and $Z^{\text {loc }}$ is the closure in $Z$ of its restriction to $\mathcal{H}_{X \times I}^{r e g}$. More generally, one could pick up a collection of subschemes of various fibers and generate inside $Z$ a local space with these particles.

(2) A local structure on a vector bundle $V$ over a local space $Z$ is a system of isomorphisms $\left.\left.\left.V\right|_{Z_{D^{\prime} \cup D^{\prime \prime}}} \cong V\right|_{Z_{D^{\prime}}} \otimes V\right|_{Z_{D^{\prime \prime}}}$. By the Segre embedding its projective bundle $\mathbb{P}(V)$ is a weakly local space. Its local part $\mathbb{P}(V)^{\text {loc }}$ is called the local projective space $\mathbb{P}^{l o c}(V)$ of a local vector bundle $V$.

4.1.2. Example: Higher loop Grassmannians for the multiplicative group. For any scheme $X$ there is a local group $\mathbb{Z}_{X}^{\text {loc }} \rightarrow \mathcal{H}_{X}$ (a group in local spaces) with fibers $\left(\mathbb{Z}_{X}^{\text {loc }}\right)_{D} \stackrel{\text { def }}{=} A_{D}$ at $D \in \mathcal{H}_{X}$. 
The related group object in factorization spaces $\mathbb{Z}^{\text {fac }} \rightarrow \mathcal{R}_{X}$ has fibers at $E \in \mathcal{R}_{X}$

$$
\left(\mathbb{Z}_{X}^{f a c}\right)_{E} \stackrel{\text { def }}{=} A_{\widehat{E}}=\lim _{\rightarrow D \in \mathcal{H}_{X}, \operatorname{supp}(D) \subseteq E}\left(\mathbb{Z}_{X}^{\text {loc }}\right)_{D}
$$

If $X$ is a smooth curve $C$ then we know that $\mathbb{Z}_{C}^{f a c}$ is the loop Grassmannian $\mathcal{G}_{C}\left(G_{m}\right)(2.2 .2)$. So, one can think of $\mathbb{Z}_{X}^{f a c}$ as the loop Grassmannian of $G_{m}$ for arbitrary schemes $X .^{(3)}$

REMARK. Loop Grassmannians of $G_{m}$ are limits of lattices. The reason is that $\mathbb{Z}_{X}^{\text {loc }} \rightarrow \mathcal{H}_{X}$ is flat. So, when a finite subset $E$ of $X$ converges to a connected finite subscheme $F$ of $X$ then the lattice $\mathcal{G}_{X}\left(G_{m}\right)_{E} \stackrel{\text { def }}{=} A_{E}=\mathbb{Z}[E]$ converges to the group ind-scheme $A_{F}=\mathcal{G}_{X}\left(G_{m}\right)_{F}$. In this way as one assembles $\widehat{c}$ by "converging a countable subset" $E$ of $X$ to a single point $c \in X$. Along the way the infinite rank lattice $\mathbb{Z}[E]$ converges to $A_{\widehat{c}}$, the (higher) loop Grassmannian of $G_{m}$.

4.1.3. Local spaces and factorization spaces. Whenever a local space $Z \rightarrow$ $\mathcal{H}_{X}$ has a growth structure $\gamma_{D^{\prime}, D}: Z_{D^{\prime}} \hookrightarrow Z_{D^{\prime \prime}}$ for $D^{\prime} \subseteq D^{\prime \prime}$ (as in 4.1.2), it defines a factorization space $Z^{f a c} \rightarrow \mathcal{R}_{X}$ with fibers

$$
Z_{E}^{f a c} \stackrel{\text { def }}{=} \lim _{\rightarrow \mathcal{H}_{X} \ni D \subseteq \widehat{E}} Z_{D}, \quad E \in \mathcal{R}_{X}
$$

So, local spaces with growth are the same as factorization spaces with $\mathcal{H}_{X^{-}}$ filtration. One may think of a local space structure on a factorization space loosely as a "nonlinear Hodge filtration".

4.1.4. Notions of local spaces. Any notion of powers of a space (see 3.1.3) comes with its own version of the formalism of local spaces. The original case used Hilbert powers as we do here. Crucially, Finkelberg noticed that one needs Cartesian powers in order to fit the Calogero-Moser spaces in the definition of local spaces. Local vector bundles for Cartesian powers include the fusion vector bundles of Feigin-Loktev ([8]).

Examples of local vector bundles for symmetric powers are the local Weyl modules of Chari-Pressley [7] (extended to the multidimensional case by Feigin-Loktev [9]). The Ito Nakamura powers are the natural setting for the Feigin-Loktev formula for fusion powers of a vector space $([9])$.

REMARK. One also has an additive notion of local spaces where product is replaced by disjoint union.

\footnotetext{
${ }^{3}$ Consider a point 0 in an affine space $\mathbb{A}^{n}$ with coordinates $x_{1}, \ldots, x_{n}$. In characteristic zero the fiber $\mathcal{G}_{X}\left(G_{m}\right)_{0}=A_{\hat{0}}$ is isomorphic to polynomials in $x_{i}{ }^{-1}$ with nilpotent coefficients. A canonical description involves the $n^{\text {th }}$ wedge power of the negative congruence subgroup.
} 
4.2. Generalization $\mathcal{G}^{P}(I, Q)$ of loop Grassmannians of reductive groups. The data here are a finite set $I$, a quadratic form $Q$ on the based lattice $\mathbb{Z}[I]$ and a finite poset $P$. We work on a smooth curve $C$ and with its simplest local space $\mathcal{H}_{C \times I}$.

4.2.1. $I$-colored local line bundles $\mathcal{L}(I, Q)$ over $C$. It is well known that a symmetric matrix $Q$ gives a local line bundle on $\mathcal{H}_{C \times I}$ by modifying the trivial line bundle along the discriminant divisors $\Delta_{i j}$ in the Hilbert scheme $\mathcal{H}_{C \times I}$

$$
\mathcal{L}(Q, I) \stackrel{\text { def }}{=} \mathcal{O}_{\mathcal{H}_{C \times I}}\left(\sum_{i \leq j} Q_{i j} \Delta_{i j}\right) .
$$

4.2.2. I-colored local vector bundles $V^{P}(I, Q)$ over $C$. We will now induce the line bundle $\mathcal{L}(I, Q)$ to a local vector bundle $V^{P}(I, Q)$ over $C$ on $\mathcal{H}_{C \times I}$ along a poset $P$. The moduli $\operatorname{Rep}_{X}(P)$ of " $X$-representations of $P$ ", is a correspondence

$$
\operatorname{Rep}_{X}(P) \underset{\subseteq}{\stackrel{\pi, \sigma}{\longrightarrow}}\left(\mathcal{H}_{C \times I}\right)^{P} \times \mathcal{H}_{C \times I} .
$$

The fiber $\sigma^{-1} D$ at $D \in \mathcal{H}_{C \times I}$ is $\operatorname{Hom}_{\text {poset }}\left(P, \mathcal{H}_{D}\right)$, where the moduli $\mathcal{H}_{D}$ of subschemes of a finite scheme $D$ is a poset for inclusions. Its elements are all systems $D^{\bullet}=\left(D^{p}\right)_{p \in P} \in\left(\mathcal{H}_{C \times I}\right)^{P}$ such that $p \leq q$ implies $D^{p} \subseteq D^{q} \subseteq D$.

This correspondence can be used to induce a family of local vector bundles $V^{p}$ on $\mathcal{H}_{C \times I}$ indexed by $p \in P$, to a single local vector bundle

$$
\operatorname{Ind}^{P}\left(V^{\bullet}\right) \stackrel{\text { def }}{=} \sigma_{*} \pi^{*}\left(\bigotimes_{p \in P} V^{p}\right) .
$$

on the same space $\mathcal{H}_{C \times I}$. The local vector bundle $\mathcal{V}^{P}(I, Q)$ on $\mathcal{H}_{C \times I}$ is obtained when all $V_{p}$ are taken to be the line bundle $\mathcal{L}(I, Q)$ :

$$
\mathcal{V}^{P}(I, Q) \stackrel{\text { def }}{=} \operatorname{Ind}^{P}(\mathcal{L}(I, Q)) \text {. }
$$

Its fiber $\mathcal{V}^{P}(I, Q)_{D}$ at $D \in \mathcal{H}_{C \times I}$ is the global sections over $\operatorname{Rep}_{X}(D)$ of the line bundle whose fiber at the point $D^{\bullet}$ is $\otimes_{p \in P} \mathcal{L}(I, Q)_{D_{p}}$.

4.2.3. The "Grassmannians" associated to a local vector bundle on $\mathcal{H}_{C \times I}$. To a local vector bundle $\mathcal{V}$ on $\mathcal{H}_{C \times I}$ we associate its zastava space $Z(\mathcal{V})$ which is the colored local projective space $\mathbb{P}^{l o c}(\mathcal{V})$ over the curve $C$.

In the case of $\mathcal{V}=V^{P}(I, Q)$ its zastava space

$$
Z^{P}(I, Q) \stackrel{\text { def }}{=} Z\left(V^{P}(I, Q)\right)=\mathbb{P}^{l o c}\left[V^{P}(I, Q)\right]
$$

has a growth structure (see 4.1.3), so we can define the corresponding semiinfinite space as the associated factorization space $S^{P}(I, Q) \stackrel{\text { def }}{=} Z^{P}(I, Q)^{\text {fac }}$. So, the fiber at $E \in \mathcal{R}_{C}$ is the limit of zastava fibers $Z^{P}(I, Q)_{D}$ as finite subschemes $D$ fill the formal neighborhood $\widehat{E}$ of $E$ in $C$

$$
S^{P}(I, Q)_{E}=\lim _{\rightarrow \mathcal{H}_{C \times I} \ni D \subseteq \widehat{E}} Z^{P}(I, Q)_{D}
$$


Finally, $\mathbb{N}[I]$ acts on $S^{P}(I, Q)$ and the corresponding loop Grassmannian is defined as

$$
\mathcal{G}^{P}(I, Q) \stackrel{\text { def }}{=} \mathbb{Z}[I] \times_{\mathbb{N}[I]} S^{P}(I, Q) .
$$

4.2.4. The case when $P$ is a point. In this case we will omit $P$ from the notation. Notice that all particles are now $\mathbb{P}^{1} .^{(4)}$ So, one is constructing $\mathcal{G}(I, Q)$ by colliding $\mathbb{P}^{1}$ 's according to a prescription given by the quadratic form $Q$.

THEOREM. Let $I$ be the set of simple coroots of an adjoint semisimple group $G$ of simply laced type. Let $Q$ is (a shift of) the basic level of $G^{(5)}$, then $\mathcal{G}^{P}(I, Q)$ is the usual loop Grassmannian $\mathcal{G}(G)$.

Remark. Similarly, when $P=[m]=\{1<\cdots<m\}$ then all particles are $\mathbb{P}^{m}$. Moreover, this $\mathbb{P}^{m}$ is naturally the $m^{\text {th }}$ symmetric power of the particle $\mathbb{P}^{1}$ for $m=1$.

4.3. Some explanations. For a torus $T=G_{m}{ }^{I}$ the loop Grassmannian $\underline{\mathcal{G}}(T)$ is obtained from the moduli $\mathcal{H}_{d}$ of infinitesimals in the disc $d$ as the abelian group $A_{d \times I}$ generated by the monoid $\mathcal{H}_{d \times I}$. We may view $\mathcal{G}(T)$ as a cloud of dust accumulated on the lattice $X_{*}(T)=\mathbb{Z}[I]$ since $\mathcal{G}(T)$ is the product of $X_{*}(T)$ and of the connected component $\mathcal{G}(T)_{0}$, but the reduced part of $\mathcal{G}(T)_{0}$ is a point. ${ }^{(6)}$

In order to assemble the loop Grassmannian $\mathcal{G}(G)$ from $\mathcal{H}_{d \times I}$ at the same time we use a quadratic form $Q$ in $X_{*}(T)$. Its geometric incarnation is a line bundle $\mathcal{L}(I, Q)$ on the space $\mathcal{H}_{d \times I}$ of infinitesimals. In terms of groups this line bundle is a restriction via $\mathcal{H}_{d \times I} \hookrightarrow \mathcal{G}(T) \subseteq \mathcal{G}(G)$ of the standard line bundle $\mathcal{O}_{\mathcal{G}(G)}(1)$ on $\mathcal{G}(G)$.

It is known that for simply laced groups the restriction under $\mathcal{G}(T) \subseteq \mathcal{G}(G)$ is an isomorphism of global sections of $\mathcal{O}_{\mathcal{G}(G)}(1)$ over $\mathcal{G}(G)$ and $\mathcal{G}(T)$. (In algebra this fact is the decomposition of the basic representation into Fock spaces.) This suggests that we should be able to describe $\mathcal{G}(G)$ in terms of the line bundle $\mathcal{L}(I, Q)$ on infinitesimals.

The way this is done by the formalism of local spaces is that one observes that certain $T$-invariant copies of $\mathbb{P}^{1}$ in $\mathcal{G}(G)$ have the properties that

(i) the restriction of $\mathcal{O}_{\mathcal{G}(G)}(1)$ to this $\mathbb{P}^{1}$ is $\mathcal{O}_{\mathbb{P}^{1}}(1)$,

(ii) $\left(\mathbb{P}^{1}\right)^{T}$ consists of two points.

(iii) $\left(\mathbb{P}^{1}\right)^{T} \subseteq \mathcal{G}(G)^{T}=\mathcal{G}(T)$ lies in $\mathcal{H}_{C \times I} \subseteq \mathcal{G}(T)$ consists of two points.

\footnotetext{
${ }^{4}$ In general for $a \in C$ and $i \in I$ the fiber $Z^{P}(I, Q)_{a i}$ is $\mathbb{P}^{l o c}\left[V^{P}(I, Q)\right]_{a i}=$ $\mathbb{P}\left[V^{P}(I, Q)_{a i}\right]$ and $V^{P}(I, Q)_{a i}$ is the vector space of sections of the line bundle $\mathcal{L}(I, Q)$ over $\operatorname{Rep}_{X}(P)_{a i}$. When $P$ is a point $\operatorname{Rep}_{X}(P)_{a i}$ is the set $\{\emptyset, a i\}$ so $V^{P}(I, Q)_{a i}$ is two dimensional.

${ }^{5}$ This is a certain $W$-invariant quadratic form on the coroot lattice.

${ }^{6}$ For a reductive $G$ the loop Grassmannian $\mathcal{G}(G)$ is reduced iff $G$ is semisimple.
} 
Such $\mathbb{P}^{1}$ can naturally be reconstructed from $\mathcal{L}(I, Q)$ since

$$
\Gamma\left[\mathbb{P}^{1}, \mathcal{O}_{\mathcal{G}(G)}(1)\right]=\Gamma\left[\mathbb{P}^{1}, \mathcal{O}_{\mathbb{P}^{1}}(1)\right]=\Gamma\left[\left(\mathbb{P}^{1}\right)^{T}, \mathcal{O}_{\mathbb{P}^{1}}(1)\right] \cong \Gamma\left[\left(\mathbb{P}^{1}\right)^{T}, \mathcal{L}(I, Q)\right]
$$

Finally one observes that these copies "generate" inside $\mathcal{G}(G)$ the zastava space from [11] and this is what is here called $Z^{P}(I, Q)$.

The "algebraic structure" on $\mathcal{G}(G)$ that one uses to generate the zastava space is the Beilinson-Drinfeld fusion on the loop Grassmannian (see 3.2). The restriction of the Beilinson-Drinfeld fusion to the zastava subspace of $\mathcal{G}(G)$ is the above locality structure on $Z^{P}(I, Q)$.

So, what we do amounts to reconstructing the zastava subspace of $\mathcal{G}(G)$ and the restrictions of the fusion structure and the line bundle $\mathcal{O}_{\mathcal{G}(G)}(1)$ to zastavas in terms of the based quadratic form $(I, Q)$.

4.3.1. From linear algebra to algebraic geometry. One of basic facts about reductive groups is their parallel existence in combinatorics (Dynkin graphs), linear algebra (lattices, cones, quadratic forms) and in algebraic geometry (algebraic groups). I would like to re-describe the passage to algebraic geometry in terms of the conjectural relative motivic cohomology.

To get from the cocharacter lattice $X_{*}(T)$ to a torus $T$, consider a point $c \stackrel{i}{\in} C$ of a smooth curve $C$. Let $C \stackrel{q}{\rightarrow}$ pt be the structure map. In terms of the conjectured functoriality of the relative motivic cohomology the dualizing sheaf $q ! \mathbb{Z}$ of $C$ should be the constant sheaf whose fiber is the classifying space $\mathbb{B}\left(G_{m}\right)$ of the multiplicative group. Then the homology of $C$ should be $q ! q ! \mathbb{Z}$. When $C$ is the formal neighborhood $\widehat{a}$ of its point $a$, this indeed says something we know, that the homology of $C$ is the compactly supported cohomology of $\mathbb{B}\left(G_{m}\right)=G_{m}[1]$, i.e., the loop Grassmannian of $G_{m}$.

So, the cocharacter lattice $X_{*}(T)$ of a torus $T$ gives $\mathbb{B}(T)$ as $i^{*} q^{!}\left(X_{*}(T)\right)$. Since this $\mathbb{B}(T)$ is a group we recover $T$ as $1 \times_{\mathbb{B}(T)} 1$. Now one can hope to get $\mathbb{B}(G)$ (and $G$ ) "mechanically" by some version of the $i^{*} q^{!}$-pull back of linear algebra data from a point via a curve. The construction of $\mathcal{G}^{P}(I, Q)$ produces directly the loop Grassmannian, i.e., it is of the form $q_{!} q^{!} \mathbb{Z}$. So, we have not displayed $\mathbb{B}(G)$ at the first step $q^{!} \mathbb{Z}$. However, this construction starts with line bundle $\mathcal{L}(I, Q)$ which indeed seems to be a pull back of type $q^{\text {! }}$ of the lattice $X_{*}(T)=\mathbb{Z}[I]=A_{I}$ and the quadratic form $Q$.

4.3.2. From combinatorics to linear algebra. The combinatorial incarnation of a simply laced group is its Dynkin graph $(I, E)$. The passage to linear algebra is the operation $A$ of forming free abelian groups. It takes a set $I$ to the lattice $\mathbb{Z}[I]=A_{I}$ and the incidence matrix $\mathcal{Q}$ (recording the number of edges) to the quadratic form $Q=A_{\mathcal{Q}}$ on $A_{I}$ (we extend the function $\mathcal{Q}: I \times I \rightarrow \mathbb{N}$ linearly to $Q: \mathbb{Z}[I] \otimes \mathbb{Z}[I] \rightarrow \mathbb{Z})$.

4.3.3. Locality equations. For a colored local vector bundle $V$ over a local space $Z$ the fiber $\mathbb{P}^{l o c}(V)_{D}$ of $\mathbb{P}^{l o c}(V)$ at a point $D \in \mathcal{H}_{C \times I}$ is a subscheme 
of the projective space $\mathbb{P}\left(V_{D}\right)$. I call the equations of $\mathbb{P}^{l o c}(V)_{D}$ in $\mathbb{P}\left(V_{D}\right)$ the locality equations at $D$.

In the case of local vector bundles $V(I, Q)$ that correspond to a group $G$, the limit of these locality equations are the equations for the standard embedding of the loop Grassmannian $\mathcal{G}(G)$ into a projective space. These are known to be equivalent (by the boson-fermion correspondence) to some standard integrable differential equations of mathematical physics, for instance for $G=S L(V)$ one gets the modified KdV hierarchies.

What is known about locality equations for data $(I, Q)$ is due to Yaping Yang. Writing these equations in general (and hopefully also for higher dimensional loop Grassmannians) is an ongoing project with Yaping Yang and Gufang Zhao. We are interested in whether we can get more integrable hierarchies in this way (and also of any possible role of the finite approximations corresponding to points $\left.D \in \mathcal{H}_{X \times I}\right)$.

\section{A CONJECTURAL RELATION OF LOOP GRASSMANNIANS AND QUIVERS}

In 5.0.1 we recall the relation of semisimple Lie algebras and quivers. In 5.0 .2 we recall the known relations between quivers and loop Grassmannians, the important one for us is the Kamnitzer-Knutson conjecture. In 5.0.3 we formulate a (conjectural) upgrade of this conjecture and finally in 5.0.4 we express a vague expectation that this upgrade is related to symplectic duality.

5.0.1. Semisimple Lie algebras and quivers. A quiver $Q$ is a directed graph i.e. a pair of sets $(I, E)$ of vertices and of directed edges connecting them. One can think of a quiver $Q$ as a way of describing the category $\mathcal{P}_{Q}$ freely generated by $Q$, the path category of $Q$. Notice that a group $\Gamma$ acting on $Q$ also acts on $\mathcal{P}_{Q}$ and so we get the stack quotient category $\mathcal{P}_{Q} / \Gamma$ which we think of as the path category of the "stack quiver" $Q / \Gamma$.

EXAMPLE. We will consider Dynkin graph of a semisimple Lie algebra $\mathfrak{g}$ as a stack graph. The usual notion of the Dynkin graph of $\mathfrak{g}$ is really a graph only when $G$ is simply laced (otherwise "vertices" have different lengths). The folding philosophy says that a multiply laced Dynkin graph $(I, E)$ should be considered as a stack quotient of a simply laced Dynkin graph $\left(I^{\prime}, E^{\prime}\right)$ by a finite group $\Gamma$ of symmetries (the length of vertices in $\left(I^{\prime}, \Gamma^{\prime}\right) / \Gamma$ now arises from stabilizers of vertices in $I^{\prime}$ ). By choosing directions of edges we get a (stack) quiver $Q=Q^{\prime} / \Gamma$ and therefore also its path category $\mathcal{P}_{Q}$. By a representation of $Q$ over $\mathbb{k}$ we mean a functor from $\mathcal{P}_{Q}$ to the category of finite dimensional vector spaces over $\mathbb{k}$.

The importance of quivers in representation theory is due to several well known ways to reconstruct the enveloping algebra and integrable representations of the Lie algebra $\mathfrak{g}$ of $G$ from the moduli of representations of the corresponding quiver $Q$, its preprojective algebra $\Pi_{Q}$ or Nakajima's double of $Q$. 
5.0.2. Quivers and loop Grassmannians. Moduli of representations of quivers and the perverse sheaves on loop Grassmannians provide two modern constructions of irreducible representations of semisimple algebraic groups that provide a new level of precision, the natural basis of representations. An advantage of the first one is that it constructs the category of representations while the second is simpler and has larger scope as it applies to arbitrary graphs. One can say that the $\mathcal{G}^{P}(I, Q)$ idea formally extends the loop Grassmannian construction to generality of arbitrary quivers. (It actually allow more inputs since the quadratic forms that we get from graphs satisfy conditions such as $Q_{i j} \geq 0$ for $i \neq j$.) However, at present it is not known what remains from the Satake mechanism in larger generality.

A substantial relation between these two constructions has been an outstanding problem, it was resolved recently by Braverman-FinkelbergNakajima [3] (see also [15]). I am particularly interested in the version of this relation conjectured earlier by Kamnitzer-Knutson. ${ }^{(7)}$

Consider a simply laced semisimple Lie algebra $\mathfrak{g}$, its adjoint group $G$ and the quiver $Q$ given by an orientation of the Dynkin graph of $\mathfrak{g}$. It is known [1] that the irreducible components $V$ of the variety of representations of the preprojective algebra $\Pi_{Q}$ of $Q$ are in a canonical bijection with certain irreducible subvarieties $X_{V}$ of the corresponding loop Grassmannian $\mathcal{G}(G)$, called $M V$-cycles [13].

The quiver Grassmannian $\operatorname{Gr}_{\Pi_{Q}}(\dot{V})$ of a representation $\dot{V}$ of the preprojective algebra $\Pi_{Q}$ is the moduli of all $\Pi_{Q}$-submodules of $\dot{V}$. More generally, for any poset $P$ one can consider the space $\operatorname{Gr}_{\Pi_{Q}}^{P}(\dot{V})$ of representations of $P$ in $\operatorname{Gr}_{\Pi_{Q}}(\dot{V})$ and Kamnitzer-Knutson considered the case when $P$ is $[m]=(1<\cdots<m)$.

When representation $\dot{V}$ is generic in the irreducible component $V$, Kamnitzer-Knutson predict a relation between its $m^{\text {th }}$ quiver Grassmannian $\operatorname{Gr}_{\Pi_{Q}}^{[m]}(\dot{V})$ and the $m^{\text {th }}$ line bundle $\mathcal{O}_{\mathcal{G}(G)}(m)$ on the loop Grassmannian $\mathcal{G}(G)$ of $G$.

Conjecture (Kamnitzer-Knutson). The following vector spaces have the same dimension: (i) the cohomology of $\operatorname{Gr}_{\Pi_{Q}}^{[m]}(\dot{V})$ and (ii) sections of $\mathcal{O}_{\mathcal{G}(G)}(m)$ over the $M V$ cycle $X_{V}$.

5.0.3. Fixed points in $M V$ cycles. Kamnitzer-Knutson were interested in upgrading this to a categorical statement. The following attempt was made possible by intense discussions with Kamnitzer-Knutson over a long period.

ConjeCtuRE. Let $\dot{V}$ be a generic representation in an irreducible component $V$ and $X_{V}$ the corresponding $M V$ cycle in $\mathcal{G}(G)$.

${ }^{7}$ While [3] considers closures of the disc group orbits, Kamnitzer-Knutson consider a larger class of $M V$-cycles. 
(a) Any poset $P$ defines a "generalized" loop Grassmannian $\mathcal{G}^{P}(G)$ of $G$. For each $M V$ cycle $X$ in $\mathcal{G}(G)$, there is a canonical subscheme $X^{P}$ of $\mathcal{G}^{P}(G)$. Then the cohomology of $\operatorname{Gr}_{\Pi_{Q}}^{P}(\dot{V})$ is the ring of functions on the subscheme $\left(\left(X_{V}\right)^{P}\right)^{T}$ of points in $\left(X_{V}\right)^{P}$ fixed by a Cartan subgroup $T$ of $G$.

(b) ["Miniscule phenomena"] For the natural line bundle $\mathcal{O}_{\mathcal{G}^{P}(G)}(1)$ on $\mathcal{G}^{P}(G)$ and any $M V$ cycle $X$ in $\mathcal{G}(G)$, the restriction of sections from $X^{P}$ to $\left(X^{P}\right)^{T}$ is an isomorphism.

(c) When $P=[m]$ then for any $M V$ cycle $X$ in $\mathcal{G}(G)$, the pairs $X^{[m]}, \mathcal{O}_{\mathcal{G}^{(m)}(G)}(1)$ and $X, \mathcal{O}_{\mathcal{G}(G)}(m)$ give the same sections

$$
\Gamma\left[X^{[m]}, \mathcal{O}_{\mathcal{G}^{(m)}(G)}(1)\right] \cong \Gamma\left[X, \mathcal{O}_{\mathcal{G}(G)}(m)\right] .
$$

REMARKS. (0) Yaping Yang and Gufang Zhao have formulated and proved a zastava version of the conjecture (a). The MV cycles are exactly the irreducible components of zastava spaces. Their version replaces a single MV cycle with the whole zastava space that contains it. On the quiver side this corresponds to degenerating the representation to zero.

(1) My student Zhijie Dong has constructed a map in one direction in the conjecture (a), from functions on the subscheme $\left(\left(X_{V}\right)^{P}\right)^{T}$ to the cohomology ring $\operatorname{Gr}_{\Pi_{Q}}^{P}(\dot{V})$ of the quiver Grassmannian.

5.0.4. Symplectic duality and Higgs-Coulomb duality. These are two conjectural frameworks for the "same" phenomenon, observed respectively in mathematics and in physics. Here, the Higgs and Coulomb branches are two irreducible components of the moduli of vacua of a given quantum field theory. A prominent case of the Higgs-Coulomb duality is the above relation of quivers and loop Grassmannians established in [3]. One aspect of the symplectic duality is the following general conjecture.

Conjecture (Hikita). When $X$ and $Y$ are symplectic dual, the cohomology of $X$ realizes the fixed locus of a torus action on $Y$ :

$$
H^{*}(X) \simeq \mathcal{O}\left(Y^{T}\right)
$$

I expect that the conjecture 5.0.3 is an example of the Hikita conjecture and therefore a manifestation of the symplectic and Higgs-Coulomb dualities. It should extend the scope of application of these dualities to the relation of quivers and loop Grassmannians from simply laced Dynkin quivers and their loop Grassmannians to all quivers and to the generalized loop Grassmannians $\mathcal{G}^{P}(I, Q)$ introduced in section 4 .

\section{LOOP GRASSMANNIANS IN ARBITRARY DIMENSION}

In section 2 we have reviewed the (Zariski aspect of) Geometric Class Field Theory. The conclusion was that the loop Grassmannian $\mathcal{G}\left(G_{m}\right)$ of the simplest reductive group $G_{m}$ can be viewed as the homology of the disc $d$ 
for a still conjectural relative motivic cohomology theory and that the (Zariski aspect of) Geometric Class Field Theory in dimension one is the Poincare duality for this relative motivic cohomology. Conjecturally such Poincare duality will exist in any dimension and we will then call it the (Zariski aspect of) general Geometric Class Field Theory.

Presently it is not known what any version of the Langlands program would mean in higher dimension. One approach to this question is to extend a well understood local geometric construction, the loop Grassmannian, to higher dimension. It seems clear how to do that for commutative groups (4.1.2). Beyond that I only have some preliminary thoughts in this direction.

The reductive groups may be too rigid for higher dimension as they have no higher cohomologies. So, one possible preliminary step (in dimension one) is section 4 where we "get rid" of the group $G$. We reconstruct the first nonabelian cohomology $\mathcal{G}(G)$ from geometry without using $G$. Instead one uses a simpler ("0-dimensional") data of a based quadratic form $(I, Q)$.

Another possible step is to construct some version of loop Grassmannians in higher dimensions. Much has been understood about two dimensional loop Grassmannians by Braverman-Finkelberg [5]. For arbitrary dimension a program has been formulated by Feigin-Loktev [8] (see also [10]). This is also the subject of an ongoing project with Yaping Yang and Gufang Zhao.

\section{REFERENCES}

[1] P. Baumann and J. Kamnitzer, Preprojective algebras and MV polytopes, arXiv: 1009. 2469.

[2] A. Beilinson and V. Drinfeld, Chiral Algebras, Colloquium Publications 51, American Mathematical Society, Providence, RI, 2004.

[3] A. Braverman, M. Finkelberg and H. Nakajima, Towards a mathematical definition of Coulomb branches of 3-dimensional $\mathcal{N}=4$ gauge theories, II, arXiv:1601.03586.

[4] R. Bezrukavnikov, M. Finkelberg and V. Schechtman, Factorizable sheaves and quantum groups, Lecture Notes in Mathematics 1691, Springer-Verlag, Berlin, 1998.

[5] A. Braverman and M. Finkelberg, Pursuing the double affine Grassmannian, part I: Transversal slices via instantons on $A_{k}$-singularities, arXiv:0711.2083; part II: Convolution, Adv. Math. 230 (2012), 414-432; part III: Convolution with affine Zastava, arXiv:1010.3499.

[6] C. E. Contou-Carrère, Corps de classes local géométrique relatif, C. R. Acad. Sci. Paris Sér. I Math. 292 (1981), 481-484.

[7] V. Chari and A. Pressley, Weyl modules for classical and quantum affine algebras, Represent. Theory 5 (2001), 191-223.

[8] B. Feigin and S. Loktev, On generalized Kostka polynomials and the quantum Verlinde rule, in: Differential topology, infinite-dimensional Lie algebras, and applications, Amer. Math. Soc. Transl. Ser. 2 194, Adv. Math. Sci. 44, Amer. Math. Soc., Providence, RI, 1999, pp. 61-79.

[9] B. Feigin and S. Loktev, Multi-dimensional Weyl modules and symmetric functions, Comm. Math. Phys. 251 (2004), 427-445.

[10] B. Feigin, A. N. Kirillov and S. Loktev, Combinatorics and geometry of higher level Weyl modules, in: Moscow Seminar on Mathematical Physics. II, Amer. Math. Soc. 
Transl. Ser. 2 221, Adv. Math. Sci. 60, Amer. Math. Soc., Providence, RI, 2007, pp. 33-47.

[11] M. Finkelberg and I. Mirković, Semiinfinite Flags. I. Case of the global curve $\mathbb{P}^{1}$, arXiv: 9707010.

[12] I. Mirković, Loop Grassmannians in the framework of local spaces over a curve, in: Recent advances in representation theory, quantum groups, algebraic geometry, and related topics, Contemp. Math. 623, Amer. Math. Soc., Providence, RI, 2014, pp. $215-226$.

[13] I. Mirković and K. Vilonen, Geometric Langlands duality and representations of algebraic groups over commutative rings, Ann. of Math. (2) 166 (2007), 95-143.

[14] D. Osipov and X. Zhu, The two-dimensional Contou-Carrère symbol and reciprocity laws, J. Algebraic Geom. 25 (2016), 703-774.

[15] B. Webster, Koszul duality between Higgs and Coulomb categories $\mathcal{O}$, arXiv: 1611.06541

\section{Neka poopćenja pojma grassmaniana petlji}

\section{Ivan Mirković}

SAŽETAK. Tema članka je pokušaj konstrukcije algebarsko geometrijskih analogona nekih topoloških ideja. Osnovni cilj je da se shemi $X$ nad komutativnim prstenom $\mathbb{k}$ pridruži njena "relativna motivska homologija", koja je opet algebarsko geometrijski objekt nad bazom $\mathbb{k}$. Ovaj projekt je motiviran teorijom brojeva i očekuje se da će Poincareova dualnost za relativnu motivsku homologiju biti algebarsko geometrijska inkarnacija Teorije polja klasa.

Department of Mathematics and Statistics

University of Massachusetts at Amherst

Amherst MA 01003-4515, USA

E-mail: mirkovic@math.umass.edu

Received: 18.5.2017. 